Check for updates

Cite this: RSC Adv., 2017, 7, 24399

\title{
In situ modified multilayer graphene toward high- performance lubricating additive
}

\begin{abstract}
Hanmin Fu, ${ }^{a}$ Xiaoqiang Fan, (D) *a Wen Li, ${ }^{a}$ Minhao Zhu, ${ }^{\text {ab }}$ Jinfang Peng ${ }^{\mathrm{b}}$ and Hao Li ${ }^{\mathrm{b}}$
To address the poor dispersibility and incompatibility of graphene toward potential applications, we propose an approach to prepare high-density modified graphene sheets by combining solvent exfoliation of graphite with chemical modification of ionic liquids (ILs). Graphite is exfoliated into graphene sheets along with in situ modification in ILs-containing solution, and ILs-modified multilayer graphene (MLG) is formed because asexfoliated graphene sheets with the charged groups of ionic liquids can undergo self-assembly together through electrostatic interaction. Here, three ILs-modified MLG was prepared using different alkyl imidazolium ILS, and their structures and tribological properties were investigated in detail. High-density coverage of IL cation and anion moieties is achieved, remarkably enhancing the dispersibility of the graphene in lubricant. MLG hybrid nanofluids show excellent friction-reducing and anti-wear ability for the contact of steel/steel because of the synergistic effect of a physical adsorption film and a tribo-chemical reaction film on the sliding surfaces.
\end{abstract}

Received 6th April 2017

Accepted 28th April 2017

DOI: $10.1039 / \mathrm{c} 7 \mathrm{ra03920d}$

rsc.li/rsc-advances

are low reactivity, poor stability and dispersity have impeded its

\section{Introduction}

Friction consumes a large portion of energy and wear causes serious loss and damage to materials, so it is of great importance to control friction and wear for energy conservation and long-term reliability. ${ }^{1,2}$ The primary method for alleviating the detrimental effects of friction is to use lubrication. With the constant consumption of lubricants over time, mechanical systems could suffer the effects of unwanted friction, excessive wear, and lubrication failure, which has been troubling us for many years. The selection of appropriate lubricants and additives for all interacting surfaces will play a significant role in reducing friction and wear, increasing service intervals and enhancing durability. ${ }^{\mathbf{1 , 3}}$ To address the challenges in friction and wear, lubrication systems must be constantly renewed and improved. Fortunately, graphene as a lubricant additive has entered people's field of vision. Graphene as a one-atom-thick $\mathrm{sp}^{2}$ carbon layered material and as mother of all graphitic nano-materials, has attracted intensive attention across academia and industry due to its impressive properties including excellent thermal conductivity, novel mechanical properties, large theoretical specific surface area compared to the in-plane value of graphite. ${ }^{4-7}$ These performance characteristics can stimulate a keen interest in widespread applications of graphene for biological sensors, super capacitors, optical devices, nano-composite materials, thermal materials, and so forth. ${ }^{8-12}$ Unfortunately, the disadvantages of graphene which

${ }^{a}$ Key Laboratory of Advanced Technologies of Materials (Ministry of Education), School of Materials Science and Engineering, Southwest Jiaotong University, Chengdu 610031, China.E-mail:fxq@home.swjtu.edu.cn; zhuminhao@swjtu.edu.cn

${ }^{b}$ Tribology Research Institute, State Key Laboratory of Traction Power, Southwest Jiaotong University, Chengdu 610031, China wide application and the further development of graphene and graphene-like materials.

Graphene is chemically insert due to its $\pi$ electrons being delocalized over the entire $2 \mathrm{D}$ network. ${ }^{13}$ In recent years, a great deal of efforts have been made to deal with these issues and expand the applications of graphene materials. Park et al. ${ }^{14,15}$ pointed out that graphene could be modified by surfactant and hydrophilic functional groups. Fan et al. ${ }^{16,17}$ proposed two approaches for the modification of graphene including the covalent combination of functional groups and the noncovalent adsorption of small molecules. Graphene with high specific surface area and surface energy allows it to readily adsorb various functional groups on the basal plane by noncovalent bond originating from weak van der Waals force or interaction of small molecules, ${ }^{15,18}$ which makes it hold a promise to keep the initial graphene structure. Covalent functionalization is attributed to a strong and irreversible bond between the functional groups and graphene. Early surface modification techniques used chemical oxidation methods to improve the dispersion and compatibility with other materials, these approaches require precise equipment with prolonged reaction time to reduce agglomerates due to the $\pi-\pi$ interaction between graphene layers, and harsh solvents such as hot nitric acid or sulfuric/nitric acid mixtures to introduce some oxygencontaining groups on the basal plane of graphene layers..$^{14,15,19,20}$ Thus, the coverage of functional groups using traditional chemical modification only happened to the location of oxygen-containing groups in graphene, which leads to the relatively poor dispersion and solubility, thereby impeding the widespread applications. 
Ionic liquids (ILs) are ionic salts in which a relatively large organic cation and a weakly coordinating inorganic anion are protected against forming a regular crystalline, thus retaining their liquid phase at a high/low temperature as the name indicates. ${ }^{21-23}$ Given the unique performance characteristics including high thermal stability, negligible low volatility, and miscibility with a range of organic compounds as well as tunable structure and performance by varying the cation or anion and their combination, ILs as "green" and versatile solvents have attracted intensive interest in synthesis, purification, chemical and catalytic reactions as well as lubrication. ${ }^{24-28}$ Tunckol et al. ${ }^{29}$ reviewed the preparation, characterization and potential application of carbon nanomaterial-ionic liquid hybrids, and enumerated the main synthetic routes of graphene-ILs and carbon nanotube-ILs. ILs have become novel candidates to modification of graphene, for example, alkyl imidazolium ILs as a class of electrolyte have been used to exfoliate graphite into multi-layer graphene (MLG)., ${ }^{\mathbf{5}, 16}$ Hence, supposing that modification of graphene occurs during the manufacturing process of the material, the coverage of functional groups can reach the optimal value on the basal plane of graphene, which will dramatically tailor the physical and chemical properties of materials.

In this study, a moderate electrochemical approach was applied to prepare three ILs-modified graphene sheets using alkyl imidazolium ILs solutions including 1-butyl-3-methyl imidazolium hexafluorophosphate (LP104), 1-hexyl-3-methyl imidazolium tetrafluoroborate (LB106), and 1-octyl-3-methyl imidazolium tetrafluoroborate (LB108). These ILs-modified graphene sheets were comprehensively investigated by surface/ interface analysis and transmission electron microscope, and their tribological properties were evaluated as a comparison with neat ILs, friction mechanism was explored by surface/ interface analysis.

\section{Experimental procedure}

\subsection{Preparation of modified multi-layers graphene by ionic liquids}

According to the previous reported literatures, ${ }^{22,23,30,31}$ three alkyl imidazolium ILs (LP104, LB106 and LB108) were synthesized, and their chemical structures are shown in Fig. 1. Highpurity graphite $(99.9 \%)$ robs were commercially obtained. The ILs-modified MLG was prepared according to our previous study. ${ }^{16}$ First, ILs solution as the electrolyte was prepared by adding $10 \mathrm{~mL}$ of ILs into $80 \mathrm{~mL}$ deionized water, then two highpurity graphite robs as anode and cathode were inserted into the electrolyte with the parallel distance of $6 \mathrm{~cm}$ depended on the diameter of beaker. Second, the applied voltage of $15 \mathrm{~V}$ was applied to the two graphite electrodes by a SMD-30 CNC double

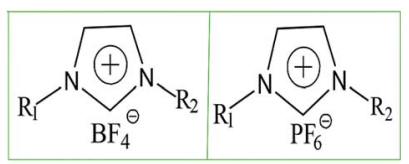

Fig. 1 Chemical structures of LB106 $\left(R_{1}=\mathrm{C}_{6} \mathrm{H}_{13}, R_{2}=\mathrm{CH}_{3}\right)$, LB108 $\left(R_{1}\right.$ $\left.=\mathrm{C}_{8} \mathrm{H}_{17}, R_{2}=\mathrm{CH}_{3}\right)$ and LP104 ILs $\left(R_{1}=\mathrm{C}_{4} \mathrm{H}_{9}, R_{2}=\mathrm{CH}_{3}\right)$. pulse power supply for an hour. As a result, the anode graphite rob was exfoliated layer by layer in the electrolyte. After chemical electrolysis, the solution became black and appeared some solid sediment at the bottom of the beaker. Then the solution was centrifuged with the speed of $3000 \mathrm{rpm}$ for 10 minutes to obtain the precipitates. Finally, the precipitates were washed with absolute ethanol and ethyl acetate several times, after drying in a vacuum oven for 24 hours at $80{ }^{\circ} \mathrm{C}$ under $0.08 \mathrm{M} \mathrm{Pa}$, the ILs-modified MLG was successfully prepared. The modified MLG sheets by LP104, LB106 and LB108 ILs were abbreviated $\mathrm{MLG}^{\mathrm{LP104-15V}}, \mathrm{MLG}^{\mathrm{LB106-15V}}$ and $\mathrm{MLG}^{\mathrm{LB108-15V}}$, respectively. In addition, the lubricants comprised of ILs and well-dispersed MLG were prepared by rotary evaporator at $80{ }^{\circ} \mathrm{C}$ for 2 hours for removing deionized water. LP104 with $\mathrm{MLG}^{\mathrm{LP104-15V}}$ was abbreviated as $\mathrm{LP} 104^{15 \mathrm{~V}}$. Similarly, LB106 with $\mathrm{MLG}^{\mathrm{LB106}-15 \mathrm{~V}}$ and LB108 with MLG ${ }^{\text {LB108-15V }}$ were abbreviated as $\mathrm{LB}_{106}{ }^{15 \mathrm{~V}}$ and LB108 ${ }^{15 V}$, respectively.

\subsection{Characterization of ILs-modified MLG}

The morphology and structure of the ILs-modified MLG were observed with JEM-2100F transmission electron microscope (HRTEM). Raman spectra were measured by Renishaw inVia Raman microscope with 532 nm laser excitation. Fourier transform infrared (FTIR) spectra were recorded in the wavenumber range of $4000-500 \mathrm{~cm}^{-1}$ with Nicolet 5700 Fourier transform infrared analysis (FTIR). The thermal stability of MLG was analyzed using a STA 449C thermo-gravimetric analyzer (TGA) at a heating rate of $10{ }^{\circ} \mathrm{C} \mathrm{min}^{-1}$ in flowing air. Lubricants with ILsmodified MLG were measured on a Shimadzu UV-2600 spectrophotometer to evaluate the dispersibility. Their functional groups of the samples were analyzed by Thermo Scientific ESCALAB 250Xi X-ray photoelectron spectroscopy (XPS) using Al-Ka radiation as the excitation source. The binding energies of the target elements were determined at a pass energy of $20.0 \mathrm{eV}$, with a resolution of about $\pm 0.3 \mathrm{eV}$, using the binding energy of contaminated carbon (C 1s: $284.8 \mathrm{eV}$ ) as the reference.

\subsection{Friction tests}

The tribological properties of lubricants were evaluated on the reciprocating UMT-2MT sliding tester at different applied loads and room temperature. The friction pairs were composed of the upper pressing AISI 52100 steel ball with standard diameter of 6 $\mathrm{mm}$ and the lower stainless steel disk. The friction tests were conducted at the applied loads of $5 \mathrm{~N}, 10 \mathrm{~N}, 15 \mathrm{~N}$ and $20 \mathrm{~N}$, sliding speed of $300 \mathrm{rpm}$, traveling distance of $5 \mathrm{~mm}$ for $60 \mathrm{~min}$. The surface contact resistance value of the lubricants during the friction process was also measured by UMT-2MT sliding tester using single-crystal $\mathrm{Si}(100)$ wafers as lower specimens. Meanwhile, the tribological behavior of lubricants under high vacuum $\left(3.2 \times 10^{-5} \mathrm{~Pa}\right)$ was investigated by a self-made rotational ball-on-disk vacuum tribometer with the applied load of 5 $\mathrm{N}$, all else being equal.

The tribological behaviors of lubricants under high applied load were evaluated on an optimal-SRV-IV reciprocation friction tester at room temperature with a ball-on-block configuration. AISI 52100 steel ball with diameter of $10 \mathrm{~mm}$, hardness $710 \mathrm{HV}$ 
as upper specimens slides reciprocally at an amplitude of $1 \mathrm{~mm}$ against the stationary lower AISI 52100 steel disk with $\Phi 24 \times$ $7.9 \mathrm{~mm}$, hardness of about $618 \mathrm{HV}$ and roughness of about $10.0 \mathrm{~nm}$. All the tests were set at the applied load of $200 \mathrm{~N}$, frequency of $25 \mathrm{~Hz}$ for $60 \mathrm{~min}$. Prior to the friction and wear test, the lubricants were added to the contact area of friction pairs. The friction coefficient was recorded automatically by a computer connected to the tester during the experimental process. After friction tests, the lower specimens were cleaned ultrasonically several times in baths of acetone and were dried with pure nitrogen for surface analysis. Each friction test was repeated three times under the same conditions in order to ensure repeatability. The wear volume of the lower specimens were measured using a MicroXAM-3D surface mapping microscope profilometer according to the cross sectional area multiplied by the length of wear track.

\subsection{Characterization and analysis}

The morphologies and element composition of wear surfaces were measured by JSM-7001F scanning electron microscope (SEM, JEOL, Japan) with Oxford-Max50 INCA-250 Energy Dispersive Spectrometer (EDS). The chemical states of typical elements on the wear surfaces were analyzed by Thermo Scientific ESCALAB 250Xi X-ray photoelectron spectroscopy (XPS) using Al-Ka irradiation as the exciting source. A pass energy of $20.0 \mathrm{eV}$ determined the binding energies of the target elements, with the resolution of around $\pm 0.3 \mathrm{eV}$, and the binding energy of carbon (C 1s: $284.8 \mathrm{eV}$ ) as the reference.

\section{Results and discussion}

\subsection{Analysis of modified multi-layers graphene}

ILs-modified MLG can be prepared by the method of solvent exfoliation of graphite in ILs solution with the assistance of an applied voltage of $15 \mathrm{~V}$. Given that the types of ILs could not only affect the efficiency of exfoliation and modification, but also determine the structure and physicochemical properties of MLG. TEM as a powerful tool was used to obtain an intuitional insight into the morphology and structure of the ILs-modified MLG. MLG ${ }^{\mathrm{LB106-15V}}, \mathrm{MLG}^{\mathrm{LB108-15V}}$ and $\mathrm{MLG}^{\mathrm{LP104-15V}}$ was characterized by TEM, and Fig. 2 provides the TEM images of the three ILs-modified MLG. Observing the image of MLG ${ }^{\mathrm{LB106}-15 \mathrm{~V}}$, it presents an obvious graphene-like structure with a smooth finish and adsorbs some ILs-capped carbon particles on the basal plane. Focusing on the TEM image of $\mathrm{MLG}^{\mathrm{LP104-15V}}$, it shows a cross-linked graphene structure with lots of wrinkles because more fluorine ions were generated by the electrolysis of LP104 and these ions dramatically enhance the electrostatic interaction of the charged graphene sheets. Keeping eyes on the TEM image of $\mathrm{MLG}^{\mathrm{LB} 108-15 \mathrm{~V}}$, it presents the relatively intact graphene structure with some overlap and curve, its high magnification TEM image shows some amorphous regions among crystalline domains because high modification density indeed induces the formation of amorphous regions, and also indicates that the ILs-modified graphene is multilayers. The result suggests that the cation and anion moieties of ILs and their physicochemical performance affect the modification density and structure of as-exfoliated graphene sheets.

Raman spectroscopy as an important analysis technique has been employed to probe into the Raman mode of graphene-like materials. For $\mathrm{sp}^{2}$ hybridized carbon such as graphene and CNTs, Raman spectroscopy can provide a great deal of information according to the characteristic peaks including $\mathrm{G}$ band (at $1580 \mathrm{~cm}^{-1}$, in-plane vibration of $\mathrm{sp}^{2}$ carbon atoms) and $2 \mathrm{D}$ band $\left(2670 \mathrm{~cm}^{-1}\right.$, two phonons double resonance Raman scattering process) and D band from introduction of defects, which can illustrate the clustering of the $\mathrm{sp}^{2}$ phase, crystal disorder, the presence of $\mathrm{sp}^{2}-\mathrm{sp}^{3}$ hybridization, defects, the introduction of chemical impurities, and so forth. ${ }^{32-34}$ Besides, the intensity ratio of $I_{2 \mathrm{D}} / I_{\mathrm{G}}$ is far more than 1 for single layer graphene with a sharp and symmetric $2 \mathrm{D}$ peak. ${ }^{35}$ Fig. $3 \mathrm{a}$ presents the Raman spectroscopy of $\mathrm{MLG}^{\mathrm{LP104-15V}}, \mathrm{MLG}^{\mathrm{LB106-15V}}$ and $\mathrm{MLG}^{\mathrm{LB108-15V}}$. It is confirmed to be multilayer graphene structure by the major Raman features $\mathrm{G}$ band $\left(\sim 1580 \mathrm{~cm}^{-1}\right)$ and $2 \mathrm{D}$ band $(\sim 2688$ $\mathrm{cm}^{-1}$ ) as well as the intensity ratio of $I_{2 \mathrm{D}} / I_{\mathrm{G}}$ (much less than 1 ). The MLG ${ }^{\mathrm{LP1} 104-15 \mathrm{~V}}$ exhibit a strong $\mathrm{D}$ peak at $1343 \mathrm{~cm}^{-1}$ due to vibrations of carbon atoms with dangling bonds at the graphene edge and wide $2 \mathrm{D}$ peak at $2688 \mathrm{~cm}^{-1}$ due to the frequent introduction of $\mathrm{sp}^{3}$-hybridized carbon atoms into the $\mathrm{sp}^{2}$-hybridized graphene layers, illustrating that moieties of ILs have adsorbed or inserted into the basal and inter-layer of graphene. Fig. $3 \mathrm{~b}$ shows the FTIR spectra of three ILs-modified MLG to confirm the presence of ILs functional groups. The characteristic peaks of $\mathrm{MLG}^{\mathrm{LP104-15V}}$ appear at $827 \mathrm{~cm}^{-1}$ and $1169 \mathrm{~cm}^{-1}$, which is due to the $\mathrm{P}-\mathrm{F}$ stretching vibration of the anion moiety and the appearance of $\mathrm{C}_{4} \mathrm{H}_{6} \mathrm{~N}_{2}$ - from LP104 ILs. The peak of MLG ${ }^{\mathrm{LB106}-15 \mathrm{~V}}$ and $\mathrm{MLG}^{\mathrm{LB108-15V}}$ at $1057 \mathrm{~cm}^{-1}$ is assigned to the B-F stretching vibration of the $\mathrm{BF}_{4}{ }^{-}$anion moieties. The $\mathrm{C}-\mathrm{N}$ band on the imidazolium ring has the characteristic peak at $1572 \mathrm{~cm}^{-1}$. The peaks at the range of $2926-3171 \mathrm{~cm}^{-1}$ are attributed to $\mathrm{C}-\mathrm{H}$ stretching of the alkyl chains on the cation moieties. ${ }^{17,19}$ The result confirms that anion and cation moieties of ILs exist in the MLG. TGA has played an important role to investigate the thermal stability of materials, Fig. 3c shows TGA curves of three ILs-modified MLG. Compared with their TGA curves, their thermal decomposition is at the temperature range from $343{ }^{\circ} \mathrm{C}$ to $384{ }^{\circ} \mathrm{C}$, while the decomposition temperature of neat ILs including LB106, LB108 and LP104 is at the temperature range from 356 to $422{ }^{\circ} \mathrm{C}$, which has been reported in the previous work..$^{16,17,19}$ With the increase of temperature, $\mathrm{MLG}^{\mathrm{LP104-15V}}$ firstly decomposed at around $343^{\circ} \mathrm{C}$ because the applied voltage of $15 \mathrm{~V}$ has caused the electrolysis of LP104, and the mass loss derives from the thermal desorption and decomposition of functional groups. LB106 and LB108 ILs-modified MLG with longer alkyl chain and $\mathrm{BF}_{4}{ }^{-}$moieties have higher thermal stability, whose mass loss is also due to the thermal decomposition of anion and cation moieties. The substantial mass loss of ILs-modified graphene accounts for $80 \%$ of the initial mass, which is due to the pyrolysis of anion and cation moieties of ILs, rather than the removal of the labile oxygen-containing functional groups, since oxygen-containing functional groups decompose at the lower temperature of around $200{ }^{\circ} \mathrm{C},{ }^{19}$ while the thermal loss of covalently bound functional groups occurs at relatively high 

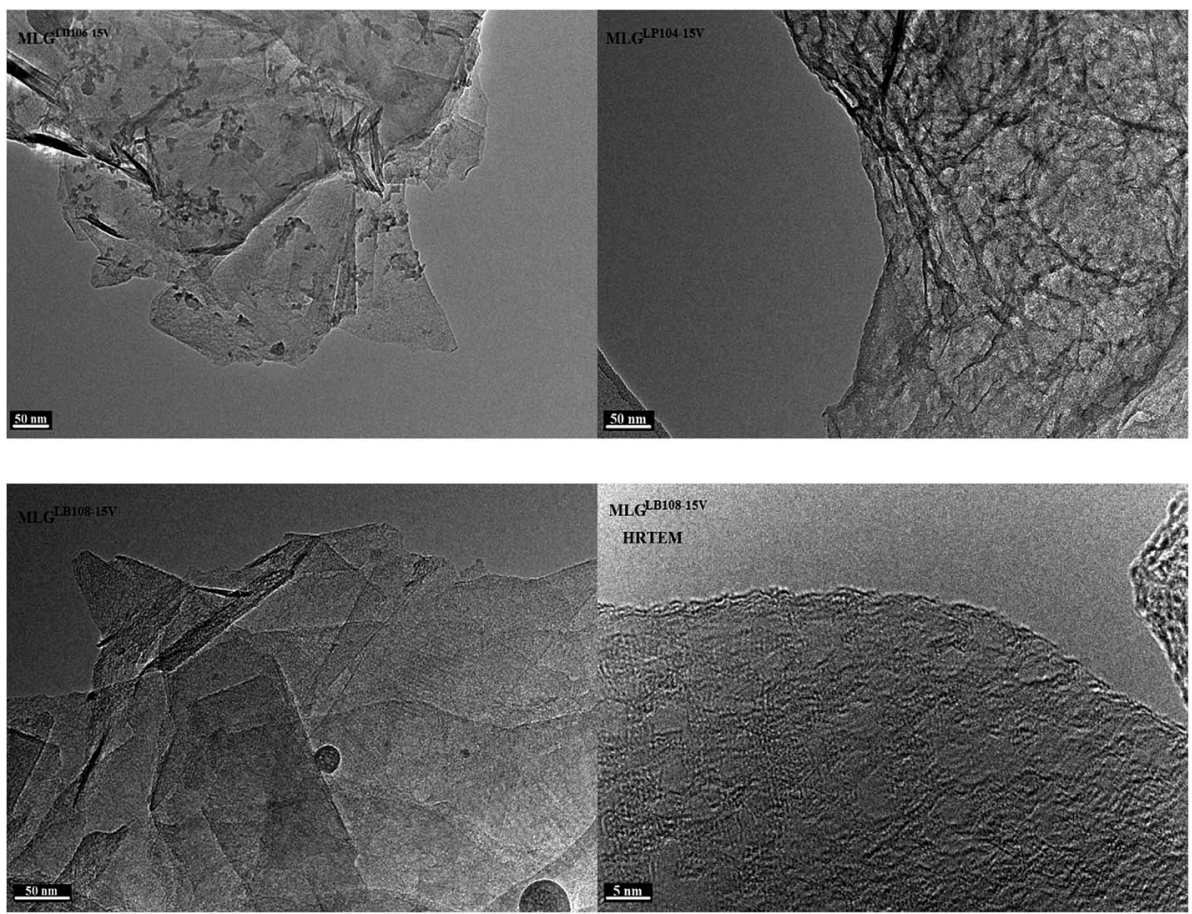

Fig. 2 TEM micrographs of ILs-modified multilayers graphene sheets including MLG ${ }^{\mathrm{LB106}-15 \mathrm{~V}}, \mathrm{MLG}^{\mathrm{LP104}-15 \mathrm{~V}}$ and MLG ${ }^{\mathrm{LB108}-15 \mathrm{~V}}$.
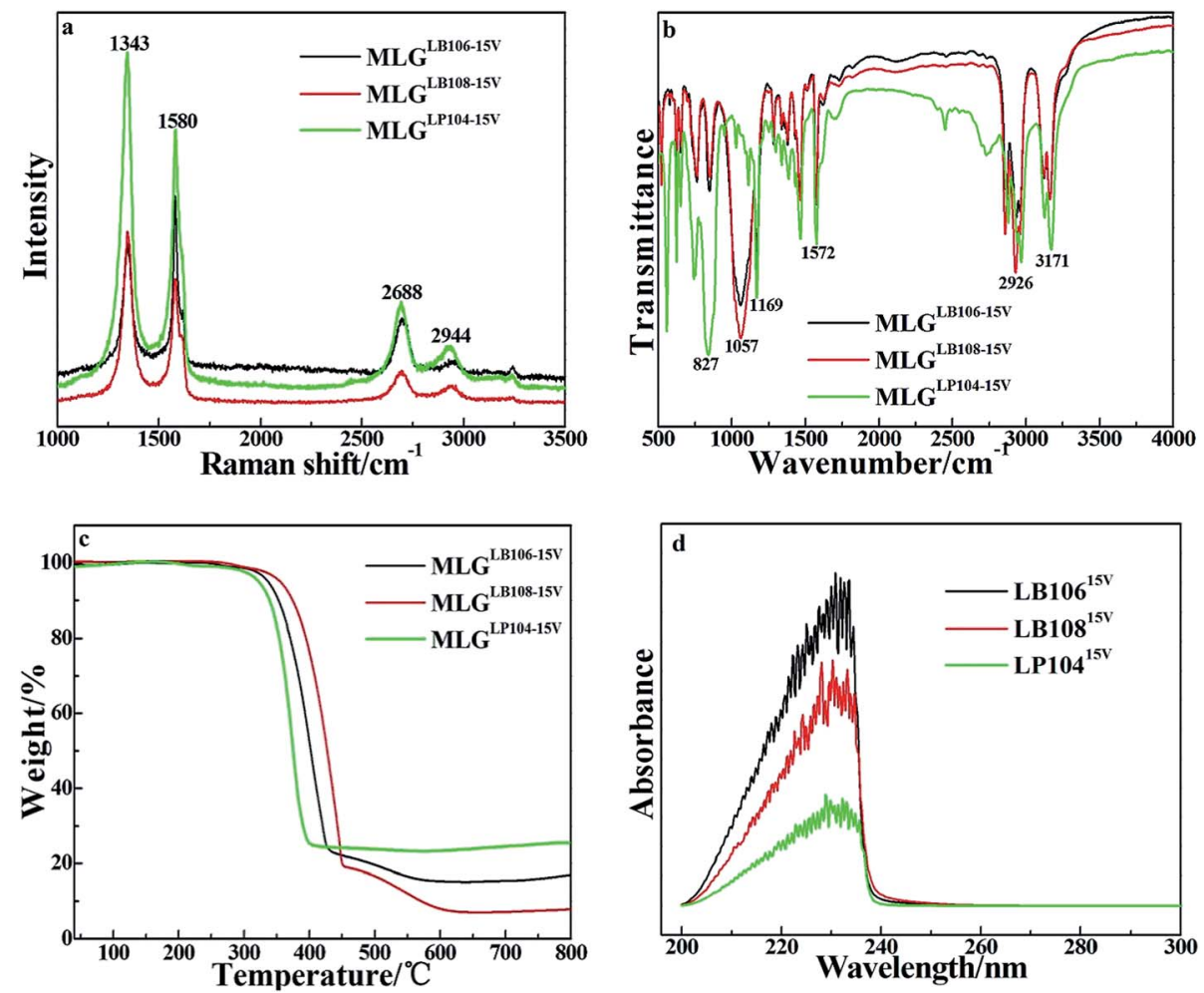

Fig. 3 Raman shift (a), FTIR spectra (b), TG curves (c) of the ILs-modified multilayer graphene (MLG ${ }^{\text {LB106-15V }}, M^{2} G^{\text {LP104-15V }}$ and MLG ${ }^{\text {LB108-15V }}$ ) and UV-Vis absorption spectra (d) of lubricants with ILs-modified MLG.

temperature range, which is consistent with the thermal decomposition temperature of neat ILs. Hence, according to the TGA curves, it is confirmed that graphene sheets have been modified in high density (about 80\%) using solvent exfoliation of graphite with chemical modification of ILs under the working potential of $15 \mathrm{~V}$. Such high density modification is realized 

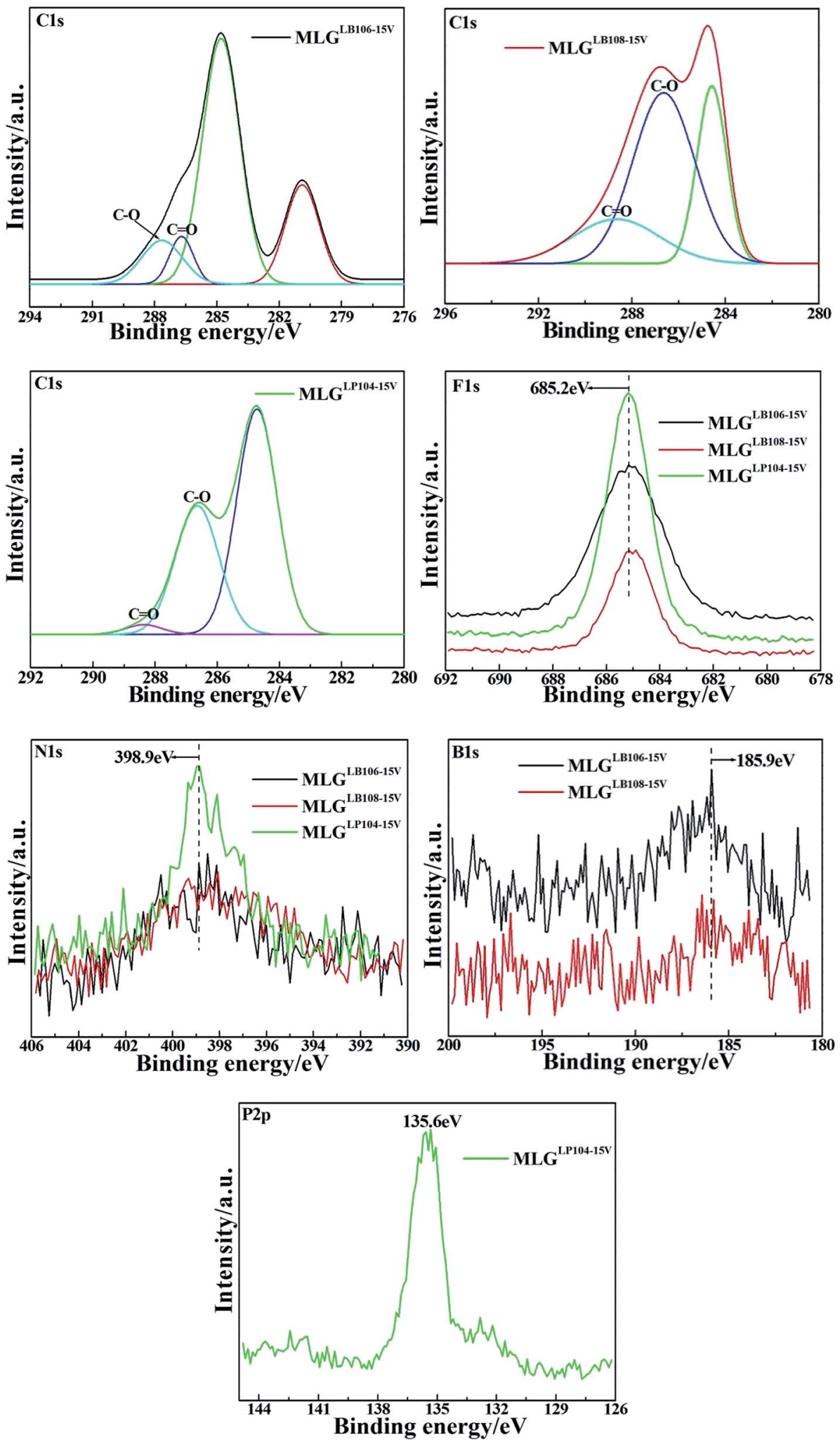

Fig. 4 X-ray photoelectron spectra of typical elements including C, F, N, B and P on the ILs-modified multilayer graphene (MLG ${ }^{\text {LB106-15V }}$, $M L G^{\mathrm{LP104}-15 \mathrm{~V}}$ and $M \mathrm{MLG}^{\mathrm{LB108-15V}}$ ).

through ILs adhering to the surface or inserting into the interlayer of graphene by chemical reaction and physical adsorption. UV-vis absorption spectroscopy was employed to gain insight into the capability of the ILs to disperse ILs-modified graphene. Fig. 3d gives the UV-vis absorption spectra of lubricants with ILsmodified MLG after storage for seven days. The UV-vis spectra of 

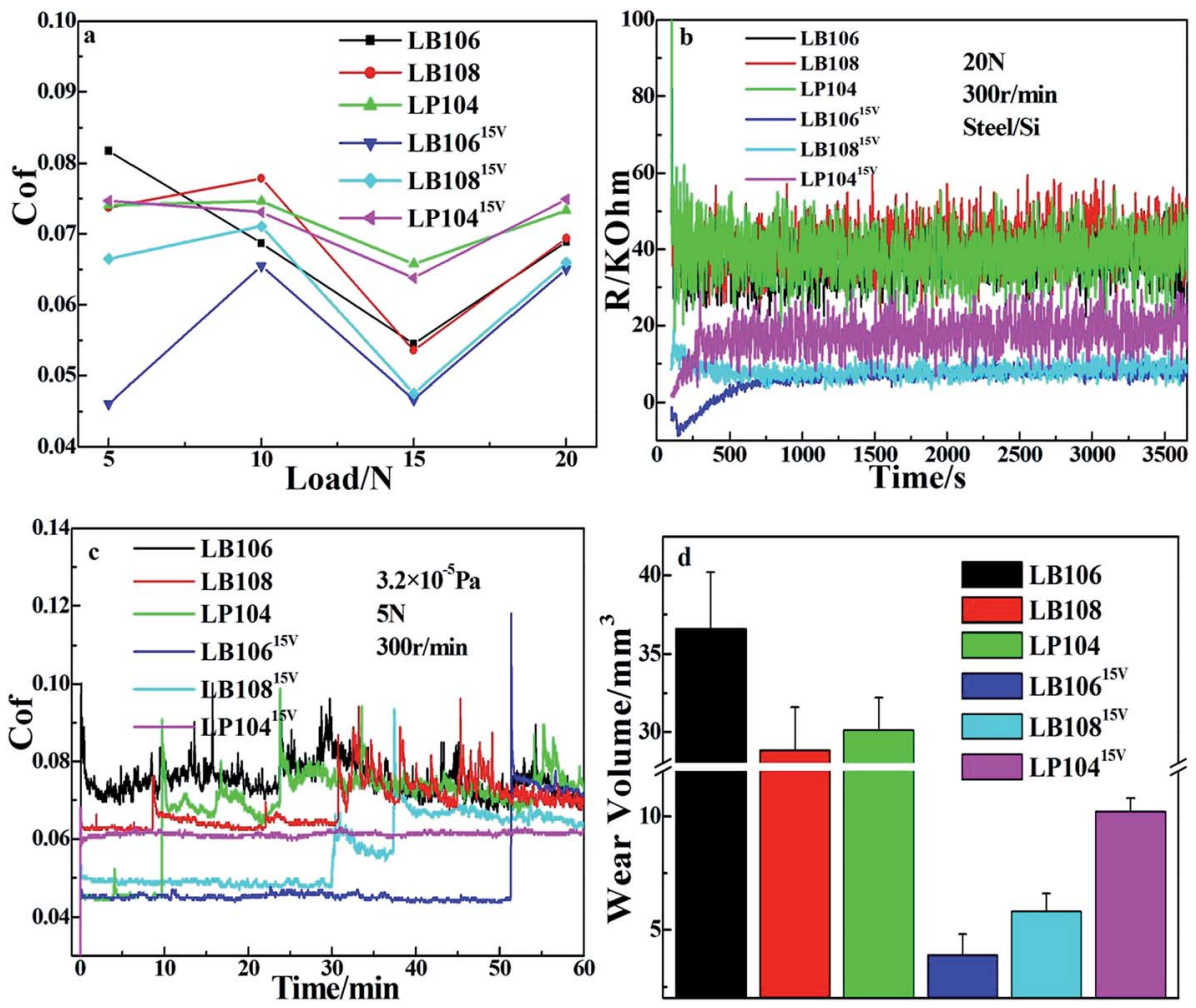

Fig. 5 Evolution of the friction coefficient (a) with the applied loads, contact resistance (b) of lubricants for the contact of steel/Si at applied load of $20 \mathrm{~N}$, friction curves (c) and wear volume (d) of lubricants under high vacuum.
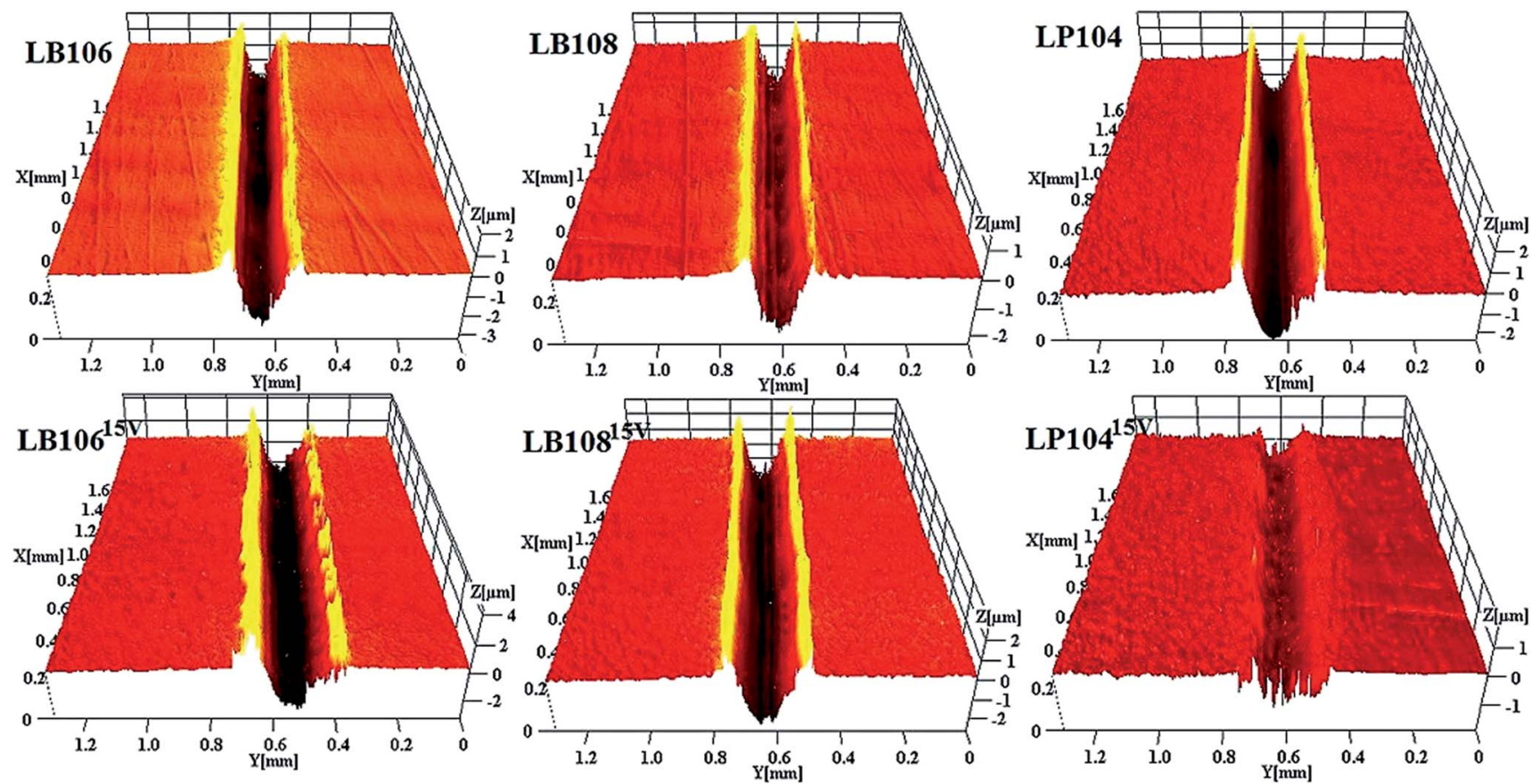

Fig. 6 3D morphologies of the worn surfaces on the steel substrates lubricated by lubricants at the applied load of $20 \mathrm{~N}$.

ILs-modified graphene using three ILs exhibit the same characteristic peaks at $231 \mathrm{~nm}$, which can be attributed to $\pi \rightarrow \pi^{*}$ transitions of aromatic $\mathrm{C}-\mathrm{C}$ bonds. ${ }^{36}$ Compared with these absorbance intensities, it is noticed that $\mathrm{LB}_{106}{ }^{15 \mathrm{~V}}$ gives the highest absorption intensity because it has the best dispersing ability, thereby dispersing the largest amount of LB106 ILs- 

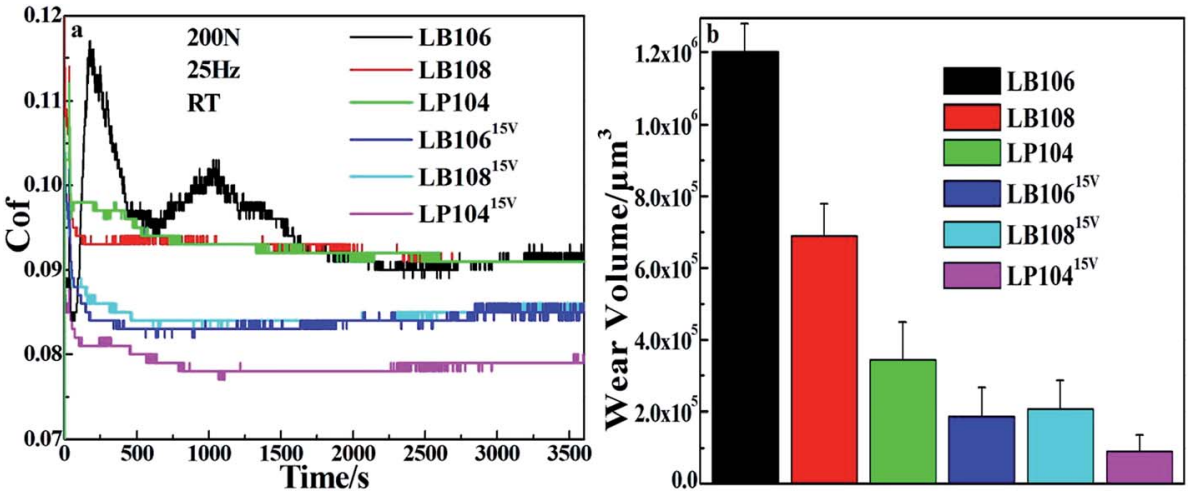

Fig. 7 Friction curves (a) and wear volume (b) of lower steel disc under lubrication of ILs with modified MLG and neat ILs at the applied load of $200 \mathrm{~N}$.
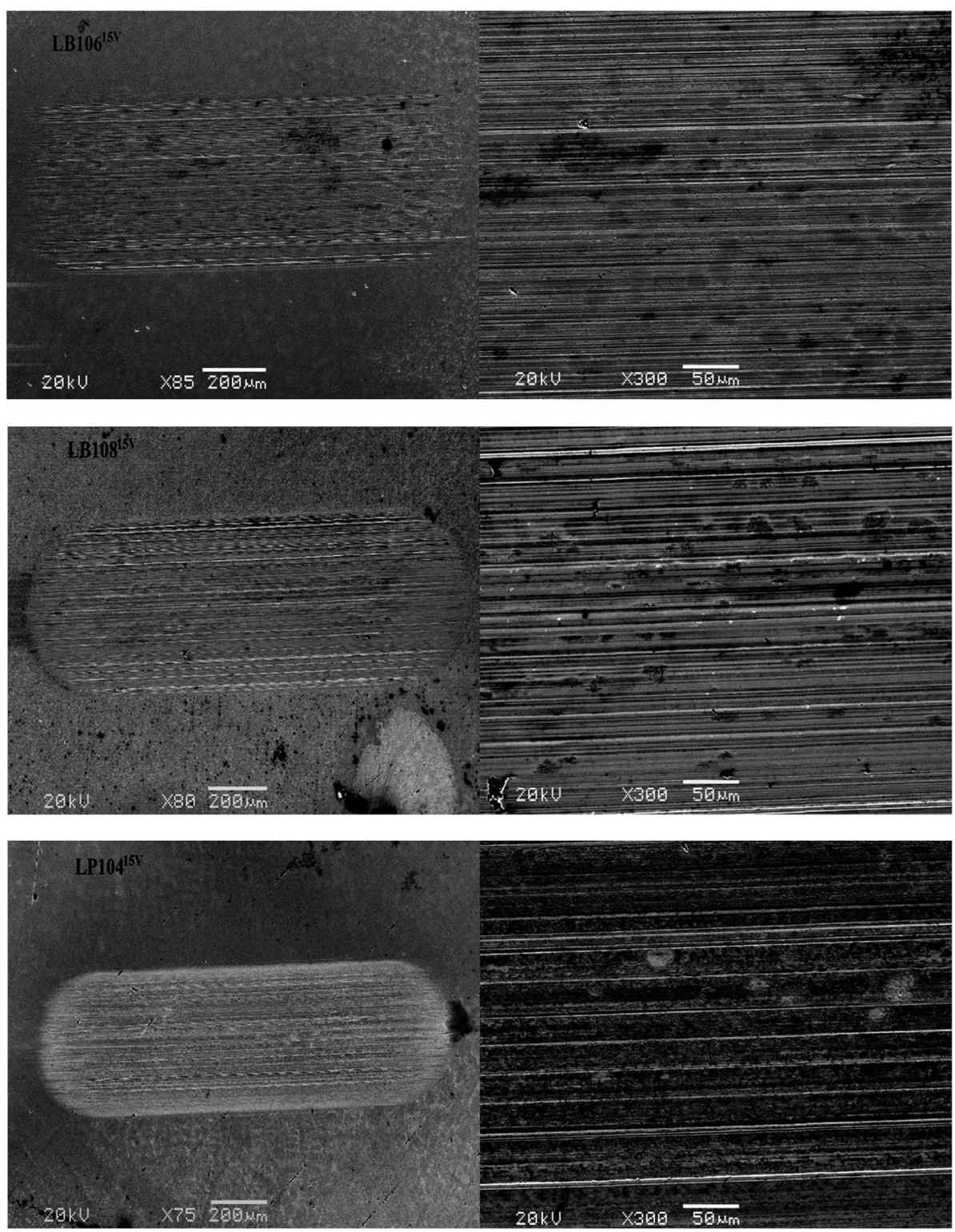

Fig. 8 SEM micrographs with low and high magnification of the worn surfaces lubricated by ILs with modified MLG at the applied load of $200 \mathrm{~N}$. 
modified graphene into the LB106. By contrast, the dispersibility of LP104 ILs-modified MLG is inferior to that of LB106 and LB108 ILs-modified MLG with long alkyl chain. So, UV-vis adsorption spectroscopy illustrates the dispersion and compatibility of ILs-modified graphene in lubricant, and the results ensure that ILs-modified graphene has long-term stability in the lubricants.

XPS spectra can provide a deeper sight into the chemical state of modified elements in the ILs-modified MLG. Fig. 4 provides the XPS spectra of typical elements on ILs. The strong and symmetrical C 1 s peak at $284.8 \mathrm{eV}$ is identified as $\mathrm{C}$ in air, using the binding energy of $284.8 \mathrm{eV}$ as the reference. The relatively weak $\mathrm{C} 1 \mathrm{~s}$ peaks appear at the binding energy of 286.8 and $288.7 \mathrm{eV}$, which is attributed to the $\mathrm{C}-\mathrm{O}$ and $\mathrm{C}=\mathrm{O}$ bonds, respectively. ${ }^{14}$ It is possible to occur the interaction of as-exfoliated graphene and oxygen atoms from the electrolysis of water due to the high applied voltage of $15 \mathrm{~V}$, which could lead to the formation of such bonds on graphene's basal planes and edges. A relatively strong $\mathrm{C}$ 1s peak of MLG $^{\text {LB106-15V }}$ locates at $280.9 \mathrm{eV}$, originating from the alkyl chains of ILs. The F 1s peaks locate at $685.2 \mathrm{eV}$, which indicates the presence of $\mathrm{F}^{-}$from the coverage of $\mathrm{BF}_{4}{ }^{-}$and $\mathrm{BF}_{6}{ }^{-}$ moieties of ILs. ${ }^{19}$ The weak $\mathrm{N}$ 1s peaks at around $398.9 \mathrm{eV}$ are assigned to $\mathrm{C}-\mathrm{N}$ bond of imidazolium ring $\left(\mathrm{C}_{3} \mathrm{H}_{4} \mathrm{~N}_{2}\right)$ of ILs. The $\mathrm{B} 1 \mathrm{~s}$ peaks at $185.9 \mathrm{eV}$ are ascribed to $\mathrm{B}-\mathrm{F}$ bond from the anion moieties of LB106 and LB108 ILs. A strong P 2p peak of $\mathrm{MLG}^{\mathrm{LP104-15V}}$ at $185.9 \mathrm{eV}$ is attributed to $\mathrm{P}-\mathrm{F}$ bond from $\mathrm{PF}_{6}{ }^{-}$ anion of LP104 ILs. ${ }^{24}$ Combined with these results of experimental characterization and analysis, it is illustrated that the anion and cation moieties of ILs including alkyl chains, imidazolium ring, $\mathrm{BF}_{4}{ }^{-}$and $\mathrm{BF}_{6}{ }^{-}$have efficiently attached to the basal planes or interlayer of graphene by physical adsorption and covalent binding, and high density modification of ILs allows the MLG to have good dispersion and compatibility in lubricant, which is favorable to enhance the tribological performance.

\subsection{Tribological properties of the lubricants}

The tribological properties of the lubricants with ILs-modified MLG (LP104 ${ }^{15 \mathrm{~V}}, \mathrm{LB} 106^{15 \mathrm{~V}}$ and LB108 ${ }^{15 \mathrm{~V}}$ ) were evaluated at the applied load of $5 \mathrm{~N}, 10 \mathrm{~N}, 15 \mathrm{~N}$ and $20 \mathrm{~N}$, with neat ILs (LP104, LB106 and LB108) as a comparison. The average friction coefficient values are shown in Fig. 5a. The $\mathrm{LB}_{106^{15 \mathrm{~V}}}$ and LB10 ${ }^{15 V}$ present lower friction coefficient value than LB106 and LB108 because the lubricants composed of ILs and ILsmodified MLG have better friction-reducing behavior, and ILsmodified MLG can provide an easy-shearing film on the sliding surfaces. Contact resistance of the lubricants during the sliding process is showed in Fig. 5b, the contact resistance reduces in the following sequence: $\mathrm{LB}_{106^{15 \mathrm{~V}}}>\mathrm{LB} 108^{15 \mathrm{~V}}>$ $\mathrm{LP} 104^{15 \mathrm{~V}}$, which provides a real-time information on the conductivity of the lubricant film. The lubricants with ILsmodified MLG display lower and more smooth contact resistance curves with small fluctuation, illustrating that ILsmodified MLG can enhance the conductivity of lubricants through the synergy of the electrical conductivity of graphene and ionic conductivity of ILs, because the ILs have high ion conductivity, while intact graphene could offer extraordinary carrier mobility. Fig. $5 \mathrm{c}$ and d show the friction curves of the
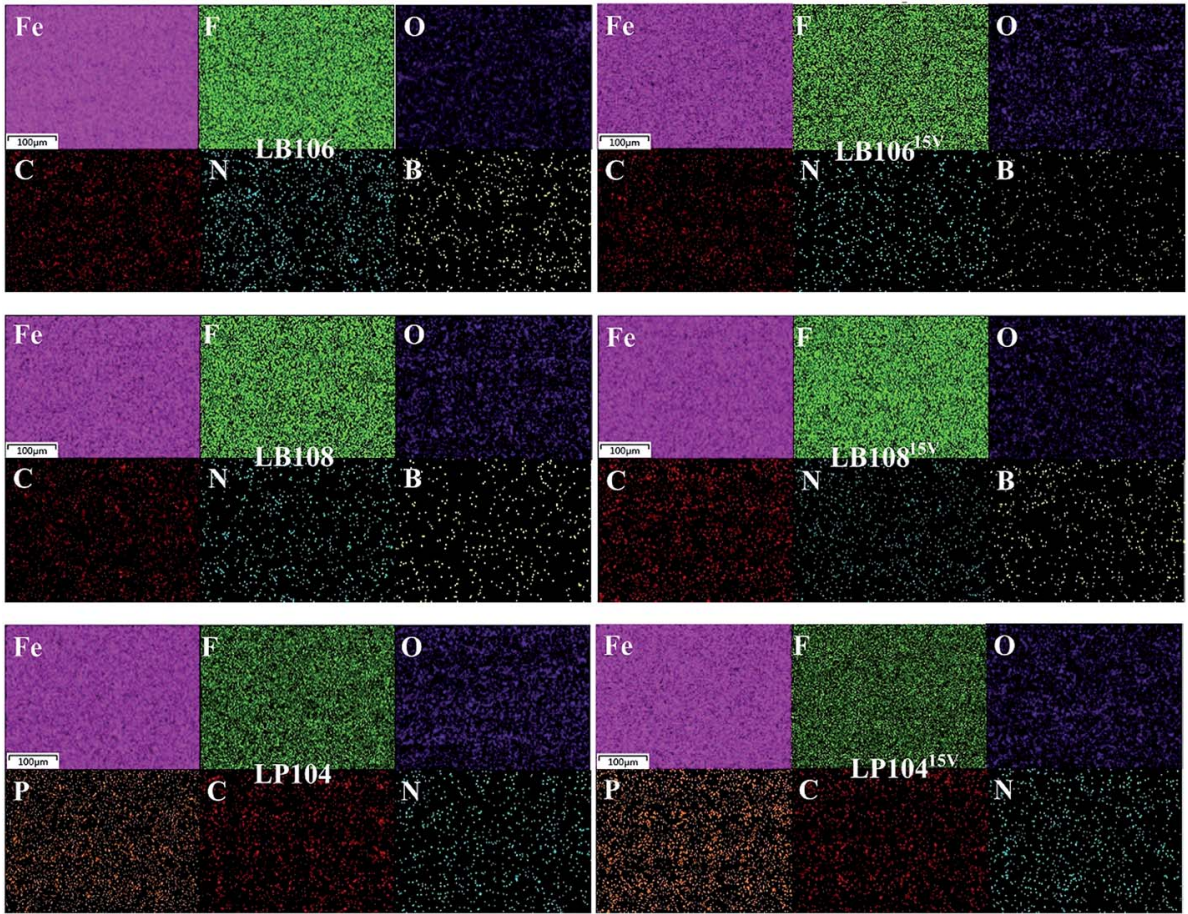

Fig. 9 EDS elemental surface distribution images of the worn surfaces lubricated by ILs with modified MLG and neat ILs at the applied load of 200 N. 
lubricants under high vacuum and the corresponding wear volume of the substrates. ILs-modified MLG can greatly enhance the friction and wear ability of lubricant, which reduces the friction coefficient, increases its stability almost without fluctuation and reduces the wear volume about 1 order of magnitude as a comparison with neat ILs. The 3D morphologies of the worn surfaces on the steel substrates are shown in Fig. 6. It can be observed that the worn surfaces lubricated by lubricants with ILs-modified MLG are relatively rough and appear obvious bulges on the sides of wear tracks in
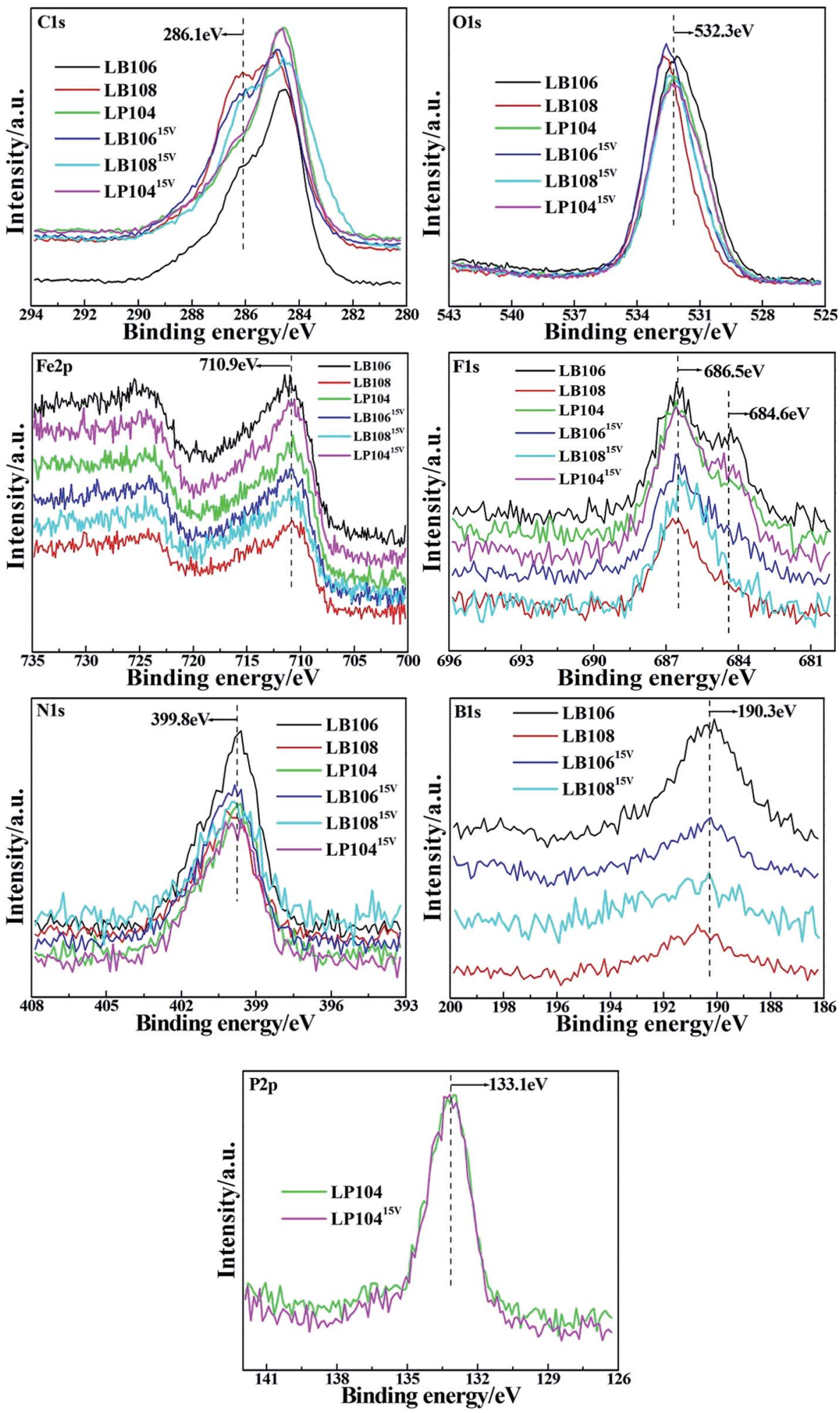

Fig. 10 XPS photoelectron spectra of typical elements on the worn surfaces lubricated by neat ILs and ILs with modified MLG. 
the sliding direction, which is attributed to the deposition of ILs-modified MLG and the interaction of functional groups and steel substrates.

Friction curve and wear volume of the lower disk lubricated by neat ILs and ILs with the modified MLG are shown in Fig. 7. Compared with their friction behaviors, it can be seen that the ILs-modified MLG has lower friction coefficients than neat ILs and the friction coefficient is more stable from starting-up and running-up stage to the end. By contrast, the friction coefficient of $\mathrm{LP} 104^{15 \mathrm{~V}}$ is the smallest (about 0.078), and the related wear volume reduces by $74 \%$, while $\mathrm{LB} 106^{15 \mathrm{~V}}$ and $\mathrm{LB} 108^{15 \mathrm{~V}}$ reduce the wear volume by $83 \%$ and $71 \%$, respectively. The results indicate that ILs-modified MLG could greatly reduce the friction and wear. So ILs-modified MLG possesses excellent tribological properties. Fig. 8 shows the SEM images of worn surfaces lubricated by ILs with modified MLG, the worn surfaces have relatively narrow wear tracks with some bulges in the sliding direction, which could be due to the formation of a protective layers derived from ILs-modified MLG. In particular, once a dense protective film was formed on the sliding surfaces, it can significantly enhance the friction-reducing and anti-wear behaviors.

\subsection{Analysis of friction mechanism}

To collect more direct evidences on the excellent tribological performance of ILs-modified MLG, EDS was used to analyze the chemical composition of worn surfaces. Fig. 9 shows the elemental distribution of the worn surfaces to provide a deeper insight into the typical elements of functional groups. It can be inferred that a dense protective layer has formed on the sliding surfaces including C, F, N, B and P from ILs and Fe from the steel substrate, suggesting that the interaction of typical elements and metal atoms has happened.

To further understand the excellent friction-reducing and anti-wear mechanism, XPS as a powerful tool was employed to investigate the chemical state of typical elements on the worn surfaces, thereby verifying the existence of any possible triboproducts. Fig. 10 presents the XPS spectra of $\mathrm{C} 1 \mathrm{~s}, \mathrm{O} 1 \mathrm{~s}, \mathrm{Fe}$ $2 \mathrm{p}, \mathrm{F} 1 \mathrm{~s}, \mathrm{~N} 1 \mathrm{~s}, \mathrm{~B} 1 \mathrm{~s}$ and $\mathrm{P} 2 \mathrm{p}$ on the worn surfaces. The $\mathrm{C} 1 \mathrm{~s}$ peaks at $286.1 \mathrm{eV}$ are ascribed to the $\mathrm{C}-\mathrm{N}$ bonding from the imidazolium ring of ILs. ${ }^{19}$ The $\mathrm{O}$ 1s peaks appearing at $532.3 \mathrm{eV}$ illustrate that the worn surfaces have complex oxide species. The Fe $2 \mathrm{p}$ peaks at $710.9 \mathrm{eV}$ and the low binding energy $\mathrm{F} 1 \mathrm{~s}$ peaks at $684.6 \mathrm{eV}$ are attributed to $\mathrm{FeF}_{2}$ due to the chemical reaction between $\mathrm{F}^{-}$of ionic liquids and Fe element during the sliding process. ${ }^{37,38}$ The high binding energy $\mathrm{F}$ 1s peaks at $686.5 \mathrm{eV}$ are assigned to the B-F and P-F bonding from the anion moieties of ILs. The $\mathrm{N} 1 \mathrm{~s}$ peaks appear at $399.8 \mathrm{eV}$, which may be identified as $\mathrm{C}-\mathrm{N}$ bonding and the transformation of nitrogen into amine. ${ }^{39,40}$ The relatively weak and wide $\mathrm{B}$ 1s peaks at $190.3 \mathrm{eV}$ ensure that $\mathrm{Fe}_{2} \mathrm{~B}$ or $\mathrm{B}_{2} \mathrm{O}_{3}$ appears on the worn surface. ${ }^{\mathbf{4 1 , 4 2}}$ The $\mathrm{P} 2 \mathrm{p}$ peaks appear at $133.1 \mathrm{eV}$, it can be sure that $\mathrm{P}-\mathrm{O}$ bonding and $\mathrm{FePO}_{4}$ have been generated on the wear tracks. ${ }^{43}$ According to XPS spectra of these typical elements, it can be confirmed that a protective film has been formed on the contact area of friction pairs during the sliding process, and the protective film is composed of physical absorption including
ILs and ILs-modified MLG, and tribo-chemical reaction products including $\mathrm{FeF}_{2}, \mathrm{Fe}_{2} \mathrm{~B}, \mathrm{FePO}_{4}$ and complex oxides on the worn surface.

Combined with the characterization and analysis of ILsmodified MLG and their tribological performance, illustrating that modification of graphene sheets is efficient and highdensity by alkyl imidazolium ILs during the process of preparation. High-density coverage of anion and cation moieties of ILs remarkably improve the dispersibility in lubricants, and is favorable to enhances the thermal stability, conductivity and tribological properties of the hybrid nanofluids originating from the physicochemical properties of graphene. So, ILsmodified MLG hybrid nanofluids show excellent frictionreducing and anti-wear ability for the contact of steel/steel at high/low applied loads or under high vacuum conditions, which mainly depends on the synergistic effect of physical adsorption film and tribo-chemical reaction film on the sliding surfaces. Liquid lubricants can afford good lubrication in the starting-up and running-in stages and mild friction conditions due to the formation of a thin oil-film on the contact area. ILs-modified MLG as additive could fill between surfaces asperities, with the increase of friction heat and mechanical force under harsh conditions, anion moieties of ILs were decomposed into active ions, and these ions can readily react with metal atoms on the steel substrates, thereby forming the anti-scratch tribochemical reaction products. So the synergy of physical absorption film of ILs and ILs-modified graphene and tribo-chemical reaction film of anti-scratch components can effectively prevent the mating surfaces from straight asperity contact, thereby significantly enhancing the tribological performance. Hence the ILs-modified MLG as an "green" advanced materials can expand the wide range of applications.

\section{Conclusions}

Intensive efforts have been made to successfully prepare ILsmodified MLG using solvent exfoliation with the assistance of applied voltage, their structure and performance characteristics were investigated in detail. The conclusions can be drawn as the following:

(a) High-density modification of graphene sheets by alkyl imidazolium ILs during the process of preparation is achieved using solvent exfoliation with the assistance of applied voltage in ILs solution.

(b) ILs-modified MLG has good dispersion and excellent friction-reducing and anti-wear abilities at coupling conditions including high/low applied loads and high vacuum.

(c) Excellent tribological behaviors of ILs-modified MLG are attributed to the synergy of physical absorption film (composed of ILs and MLG) and tribo-chemical reaction film (comprised of fluoride, phosphate and complex oxides) on the sliding surfaces.

\section{Acknowledgements}

The authors gratefully acknowledge the financial support provided by China National Funds for Distinguished Young 
Scientists (No. 51025519), the Changjiang Scholarships and Innovation Team Development Plan (No. IRT1178), the National Natural Science Foundation of China (No. 51627806).

\section{References}

1 K. Holmberg, P. Andersson and A. Erdemir, Tribol. Int., 2012, 47, 221-234.

2 M. G. Fan, Y. M. Liang, F. Zhou and W. M. Liu, RSC Adv., 2012, 2, 6824-6830.

3 A. Somers, B. Khemchandani, P. C. Howlett, J. Z. Sun, D. R. Macfarlane and M. Forsyth, ACS Appl. Mater. Interfaces, 2013, 5, 11544-11553.

4 A. K. Geim, Science, 2009, 324, 1530-1534.

5 N. Liu, F. Luo, H. X. Wu, Y. H. Liu, C. Zhang and J. Chen, Adv. Funct. Mater., 2008, 18, 1518-1525.

6 J. F. Wang, M. Y. Zhu, R. A. Outlaw, X. Zhao, D. M. Manos, B. C. Holloway and V. P. Mammana, Appl. Phys. Lett., 2004, 85, 1265-1267.

7 K. S. Novoselov, A. K. Geim, S. V. Morozov, D. Jiang, Y. Zhang, S. V. Dubonos, I. V. Grigorieva and A. A. Firsov, Science, 2004, 306, 666-669.

8 S. Stankovich, D. A. Dikin, G. H. B. Dommett, K. M. Kohlhaas, Z. J. Zimney, E. A. Stach, R. D. Piner, S. T. Nguyen and R. S. Ruoff, Nature, 2006, 442, 282-286.

9 T. Y. Kim, H. W. Lee, M. D. Stoller, D. R. Dreyer, C. W. Bielawski, R. S. Ruoff and K. S. Suh, ACS Nano, 2011, 5, 436-442.

10 C. S. Shan, H. F. Yang, J. X. Song, D. X. Han, A. Ivaska and L. Niu, Anal. Chem., 2009, 81, 2378-2382.

11 H. C. Lee, W. W. Liu, S. P. Chai, A. R. Mohamed, A. Aziz, C. S. Khe, N. M. S. Hidayah and U. Hashim, RSC Adv., 2017, 7, 15644-15693.

12 Y. Chen, X. Zhang, D. F. Zhang, P. Yu and Y. W. Ma, Carbon, 2011, 49, 573-580.

13 M. Quintana, E. Vazquez and M. Prato, Acc. Chem. Res., 2013, 46, 138-148.

14 S. Park, K. S. Lee, G. Bozoklu, W. W. Cai, S. T. Nguyen and R. S. Ruoff, ACS Nano, 2008, 2, 572-578.

15 J. Park and M. D. Yan, Acc. Chem. Res., 2013, 46, 181-189. 16 X. Q. Fan, L. P. Wang and W. Li, Tribol. Lett., 2015, 58, 12. 17 X. Q. Fan and L. P. Wang, Tribol. Int., 2015, 88, 179-188.

18 S. Park, A. N. Jinho, R. D. Piner, I. Jung, D. X. Yang, A. Velamakanni, S. T. Nguyen and R. S. Ruoff, Chem. Mater., 2008, 20, 6592-6594.

19 X. Q. Fan and L. P. Wang, J. Colloid Interface Sci., 2015, 452, 98-108.
20 S. Ryu, M. Y. Han, J. Maultzsch, T. F. Heinz, P. Kim, M. L. Steigerwald and L. E. Brus, Nano Lett., 2008, 8, 45974602.

21 J. Qu, J. J. Truhan, S. Dai, H. M. Luo and P. J. Blau, Tribol. Lett., 2006, 22, 207-214.

22 R. Hagiwara and Y. Ito, J. Fluorine Chem., 2000, 105, 221-227.

23 P. Bonhote, A. Dias, N. Papageorgiou, K. Kalyanasundaram and M. Gratzel, Inorg. Chem., 1996, 35, 1168-1178.

24 L. L. Zhang, J. B. Pu, L. P. Wang and Q. J. Xue, Carbon, 2014, 80, 734-745.

25 F. Zhou, Y. M. Liang and W. M. Liu, Chem. Soc. Rev., 2009, 38, 2590-2599.

26 R. Gusain and O. P. Khatri, RSC Adv., 2016, 6, 3462-3469.

27 K. Goel, S. Bera, M. Singh and D. Mondal, $R S C A d v$. , 2016, 6, 106806-106820.

28 M. G. Freire, P. J. Carvalho, A. M. Fernandes, I. M. Marrucho, A. J. Queimada and J. A. P. Coutinho, J. Colloid Interface Sci., 2007, 314, 621-630.

29 M. Tunckol, J. Durand and P. Serp, Carbon, 2012, 50, 43034334.

30 J. D. Holbrey, W. M. Reichert, R. P. Swatloski, G. A. Broker, W. R. Pitner, K. R. Seddon and R. D. Rogers, Green Chem., 2002, 4, 407-413.

31 T. Welton, Chem. Rev., 1999, 99, 2071-2083.

32 M. S. Dresselhaus, A. Jorio, M. Hofmann, G. Dresselhaus and R. Satio, Nano Lett., 2010, 10, 751-758.

33 J. S. Park, A. Reina, R. Saito, J. A. Kong, G. Dresselhaus and M. S. Dresselhaus, Carbon, 2009, 47, 1303-1310.

34 M. S. Dresselhaus, G. Dresselhaus, R. Saito and A. Jorio, Phys. Rep., 2005, 409, 47-99.

35 C. Thomsen and S. Reich, Phys. Rev. Lett., 2000, 85, 52145217.

36 D. Konios, M. M. Stylianakis, E. Stratakis and E. Kymakis, J. Colloid Interface Sci., 2014, 430, 108-112.

37 J. Q. Yang, Y. Liu, Z. Y. Ye, D. Z. Yang and S. Y. He, Tribol. Lett., 2010, 40, 139-147.

38 Z. Y. Wang, Y. Q. Xia, Z. L. Liu and Z. Z. Wen, Tribol. Lett., 2012, 46, 33-42.

39 M. R. Cai, Z. Zhao, Y. M. Liang, F. Zhou and W. M. Liu, Tribol. Lett., 2010, 40, 215-224.

40 H. Z. Wang, Q. M. Lu, C. F. Ye, W. M. Liu and Z. J. Cui, Wear, 2004, 256, 44-48.

41 G. Mavel, J. Escard, P. L. D. Costa and J. Castaing, Surf. Sci., 1973, 35, 109-116.

42 H. B. Zhang, Y. Q. Xia, M. Yao, Z. F. Jia and Z. L. Liu, Tribol. Lett., 2009, 36, 105-111.

43 I. Minami, Molecules, 2009, 14, 2286-2305. 